\title{
Spinal Epidural Myeloid Sarcoma with Paraplegia in Acute Myeloid Leukemia Treated with Radiation and Chemotherapy: A Case Report
}

\section{Chiaki Sato, Naohisa Miyakoshi*, Michio Hongo, Yuji Kasukawa, Yoshinori Ishikawa, Daisuke Kudo, Yoichi Shimada}

Department of Orthopedic Surgery, Akita University Graduate School of Medicine, Akita, Japan

Email: *miyakosh@doc.med.akita-u.ac.jp

How to cite this paper: Sato, C., Miyakoshi, N., Hongo, M., Kasukawa, Y., Ishikawa, Y., Kudo, D., and Shimada, Y. (2019) Spinal Epidural Myeloid Sarcoma with Paraplegia in Acute Myeloid Leukemia Treated with Radiation and Chemotherapy: A Case Report. Open Journal of Orthopedics, 9, 131-136.

https://doi.org/10.4236/ojo.2019.96013

Received: May 21, 2019

Accepted: June 27, 2019

Published: June 30, 2019

Copyright $\odot 2019$ by author(s) and Scientific Research Publishing Inc. This work is licensed under the Creative Commons Attribution International License (CC BY 4.0).

http://creativecommons.org/licenses/by/4.0/

\begin{abstract}
Myeloid sarcoma occurs in 1\% - 9\% of patients with myelogenous leukemia. Spinal epidural myeloid sarcoma is particularly rare, and its treatment has not been established. A 27-year-old woman complained of pain on her left chest, back around the scapula, and neck. Magnetic resonance imaging (MRI) showed a thoracic epidural tumor. One week after her visit, she developed motor weakness of her lower extremities and dysuria, and she was diagnosed with acute myelogenous leukemia (AML) on peripheral blood analysis. The epidural tumor was strongly suspected to be myeloid sarcoma. The paralysis of the lower extremities and bladder dysfunction were not progressive, and chemotherapy and local radiation therapy to the spine were performed. Improvement of paralysis and complete reduction of tumor volume were achieved by the combination of local low-dose radiation therapy and chemotherapy.
\end{abstract}

\section{Keywords}

Myeloid Sarcoma, Acute Myeloid Leukemia, Spinal Cord Compression

\section{Introduction}

Myeloid sarcoma, also known as granulocytic sarcoma or chloroma, is an extramedullary localized tumor of immature myeloid cells in patients with acute myeloid leukemia (AML), chronic myeloid leukemia, and myelodysplastic syndrome [1]. It has been reported that myeloid sarcoma occurs in $1 \%-9 \%$ of patients with myelogenous leukemia [2] [3] [4]. The presence of myeloid sarcoma 
adversely affected the relapse and disease-free survival rates in adult patients with AML [5]. Commonly involved sites of myeloid sarcoma are the skin, soft tissue, bone, periosteum, and lymph nodes [2] [6] [7]. Spinal epidural myeloid sarcoma is not common [7], and there are several reports of spinal cord compression with paralysis [8]-[14]. The treatment for such patients with neurological symptoms varies, including surgery, radiation therapy, and chemotherapy. However, the treatment protocol has not been established. A case of AML, which showed an acute onset of myelopathy due to spinal epidural myeloid sarcoma at the thoracic spine and was treated with local low-dose radiation therapy and chemotherapy, is presented.

\section{Case Report}

A 27-year-old woman complained of a 2-month history of pain of her left chest, back around the scapula, and neck. Magnetic resonance imaging (MRI) ordered by a primary care physician showed an epidural tumor at T3-5. She developed a motor weakness of her lower extremities and dysuria one week after the MRI. She was referred to a general hospital, and leukemia was suspected based on a complete blood count. She was immediately referred to our hospital and admitted to the hematology department. Although she could walk with a cane, she had paresthesia from three fingers below the sternoclavicular joints and motor weakness of the extensor hallucis longus muscles (manual muscle test grade 4/5) with hyperreflexia of both lower extremities, positive ankle clonus, and dysuria. The white blood cell count was $9000 / \mu$ l (blast cells accounted for $23 \%$ ), the he-

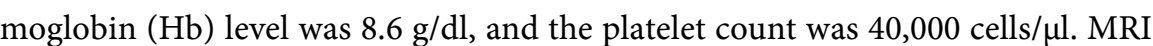
showed a left dorsal epidural tumor at T3-T5 with iso-intensity on T1 and T2-weighted signal images (Figure 1(a) and Figure 1(b)). T2-weighted axial images demonstrated an epidural tumor compressing the spinal cord at the T4 level (Figure 1(c)). Computed tomography (CT) showed an epidural soft tissue tumor at the T3-T5 level. There was no mechanical scalloping effect on the surrounding bone (not shown). Since the hematologic study from peripheral blood resulted in the diagnosis of AML and she had been healthy, the epidural tumor was strongly suspected to be myeloid sarcoma.

Followed by the careful interview of her history of paralysis and physical examination, we have evaluated that the paralysis of the lower extremities and bladder dysfunction were not progressive. Then, intravenous methylprednisolone pulse treatment and local radiation therapy to the spine were started on the day of admission to our hospital instead of emergent surgical decompression. On the day following irradiation, the subjective symptoms of paralysis and paresthesia were improved. On the third day after initiating irradiation, the AML type was diagnosed as M2 by the French-American-British (FAB) Classification with a blast ratio of $70 \%$ by bone marrow aspiration. Then, remission induction therapy with 5 days of daunorubicin and 7 days of cytarabine (DNR/Ara-C) was also started. On the fifth day, MRI showed that the dorsal epidural tumor had va 


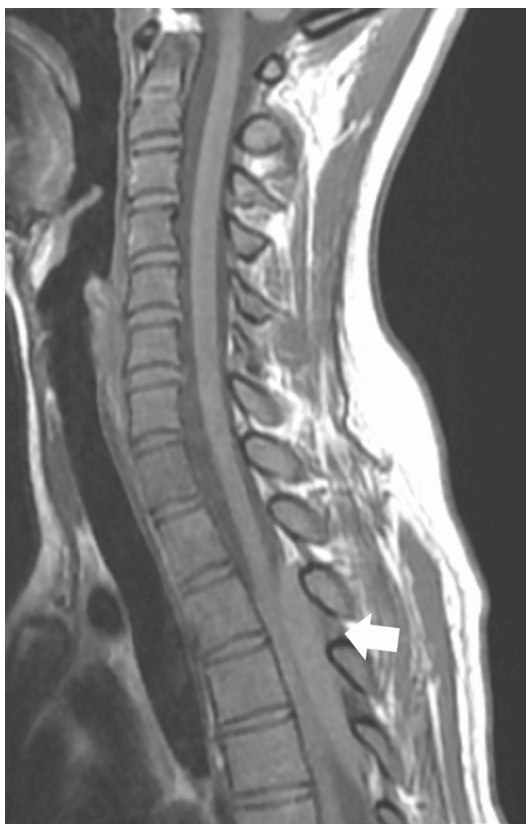

(a)

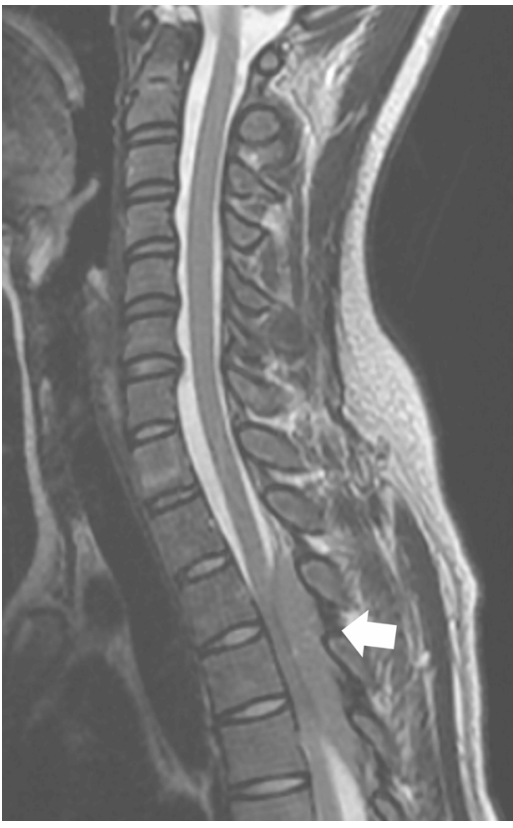

(b)

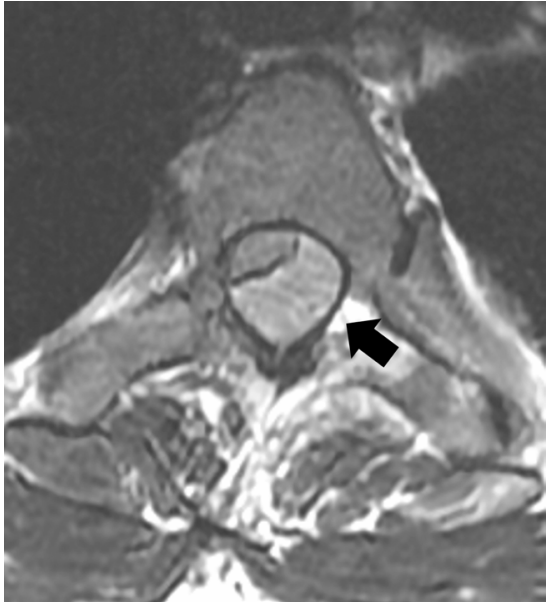

(c)

Figure 1. Magnetic resonance imaging (MRI) on admission. (a) Sagittal T1-weighted image showing a left dorsal epidural iso-intensity mass at T3-5 (arrow), and (b) sagittal T2-weighted image showing hypo-intensity (arrow). (c) Axial T2-weighted image at T4 showing a left-sided epidural mass (arrow) compressing the spinal cord.

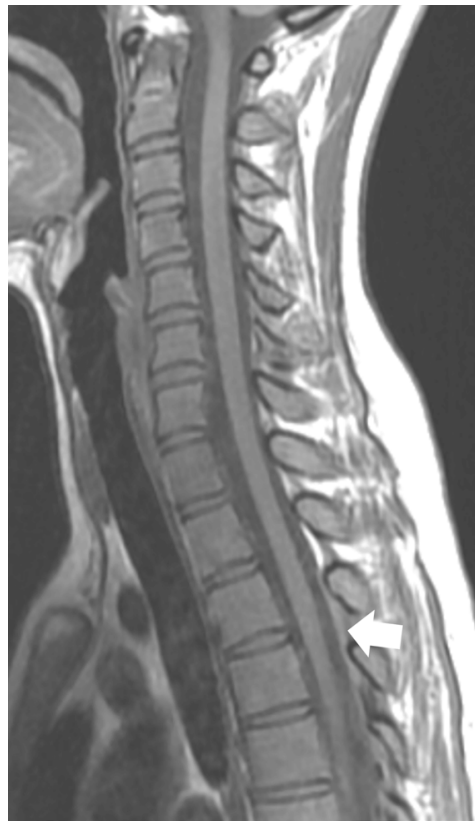

(a)

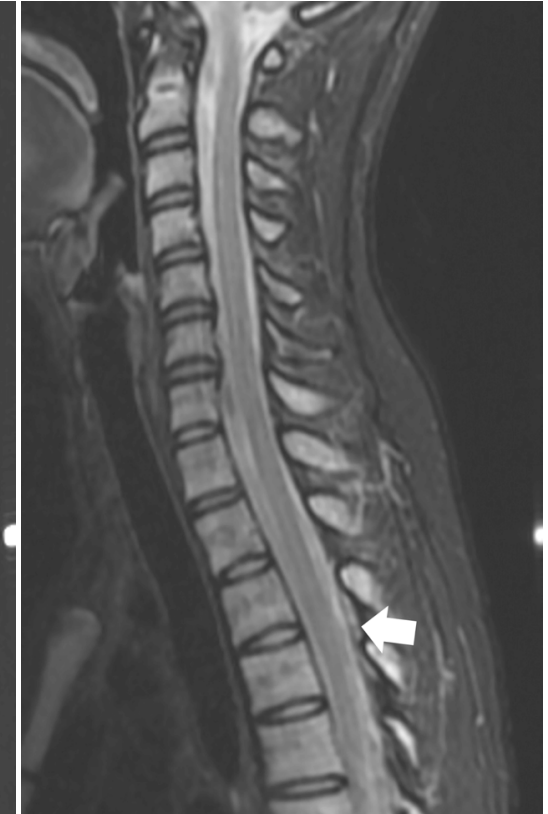

(b)

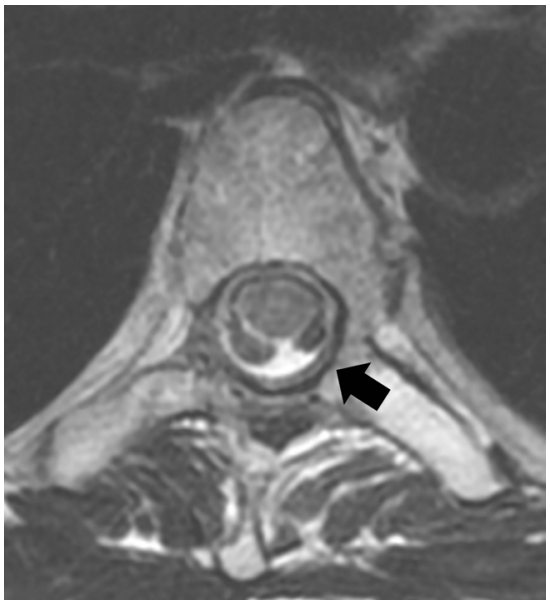

(c)

Figure 2. MRI on the fifth day of irradiation. Sagittal T-weighted (a) and T2-weighted (b) MRI showing that the mass in the spinal epidural space (arrows) has vanished. (c) Axial T2-weighted image at T4 showing complete disappearance of the mass and the cord compression (arrow).

nished (Figures 2(a)-(c)), and the muscle strength of the lower extremity became normal. On the seventh day, the neurological symptoms improved further, and thus the irradiation was stopped with a total of $21 \mathrm{~Gy}$. Although paresthesia on the front of the thigh remained, the patient was able to walk completely on her 
own. Thereafter, the patient achieved complete remission, with continuing chemotherapy for consolidation therapy with high-dose cytarabine for 2 months. One month after final chemotherapy, she underwent allogeneic stem cell transplantation. Two years post-irradiation, no recurrence of neurological deficit or paralysis has been observed.

\section{Discussion}

There have been several case reports and small case series of spinal myeloid sarcoma with spinal cord compression causing paralysis [8]-[14]. The majority of the patients with neurological symptoms were treated with surgical decompression combined with chemotherapy [8] [10] [11] [14], radiation therapy [9], and both radiation and chemotherapy [12]. However, no specific guidelines on the management of myeloid sarcoma associated with AML are currently available.

Surgical decompression followed by radiotherapy has been reported to be more effective than radiotherapy alone in terms of pain control and ambulation for spinal cord compression by metastatic disease [15]. However, this study excluded patients with radiosensitive tumors such as leukemia and lymphoma. Radiation therapy for spinal myeloid sarcoma has been reported to be effective [7] [16]. The tumor volume in all patients regressed completely or partially after radiation therapy [16]. Motor function of the lower extremity improved gradually with radiation [9]. As radiation therapy for local control of myeloid sarcoma, Bakst et al. recommended a protocol for a localized mass with irradiation of $24 \mathrm{~Gy}$ in 12 divided doses [7]. In the current case, compression of the spinal cord due to the mass of the vertebral canal was observed on MRI, but paralysis and bladder dysfunction were not severe and progressive. Therefore, local radiation therapy and chemotherapy for remission induction therapy were chosen. The size of the epidural mass was significantly decreased in a short time, and the lower extremity symptoms improved. Considering the possibility of total body irradiation for remission induction and the spinal cord tolerable dose at recurrence, radiation therapy was stopped at a total dose of $21 \mathrm{~Gy}$ on the seventh day of irradiation.

Myeloid sarcoma is a local lesion of leukemia, so remission-induction chemotherapy is considered necessary for radical treatment. Due to the high rate of progression to acute leukemia, especially in patients who are treated with localized methods, conventional systemic treatments are recommended for the treatment of isolated myeloid sarcoma presenting concomitantly with AML [17] [18]. Since the current case was diagnosed as AML on bone marrow aspiration, it was essential to give chemotherapy concurrently with localized radiotherapy.

A limitation of this case report was that there was no pathological evidence of the local lesion by excisional biopsy. However, the use of radiation therapy and adjuvant chemotherapy for the lesion relieved the neurological symptoms and reduced the size of the mass. Early and precise diagnosis with imaging studies followed by analyses of peripheral blood and bone marrow aspiration led to the 
appropriate treatment without surgical intervention.

\section{Conclusion}

The improvement of paralysis and complete reduction of tumor volume was achieved by the combination of local low-dose radiation therapy and chemotherapy for a case of spinal epidural myeloid sarcoma in an AML patient. Chemotherapy based on AML treatment is recommended for myeloid sarcoma, but it is also reported that low-dose radiation therapy is effective for local control.

\section{Ethical Considerations}

The authors report no conflict of interest. The informed consent of the patient was obtained prior to the use of the data, and confidentiality was assured.

\section{Conflicts of Interest}

The authors declare no conflicts of interest regarding the publication of this paper.

\section{References}

[1] Pileri, S.A., Ascani, S., Cox, M.C., Campidelli, C., Bacci, F., Piccioli, M., Piccaluga, P.P., Agostinelli, C., Asioli, S., Novero, D., Bisceglia, M., Ponzoni, M., Gentile, A., Rinaldi, P., Franco, V., Vincelli, D., Pileri Jr., A., Gasbarra, R., Falini, B., Zinzani, P.L. and Baccarani, M. (2007) Myeloid Sarcoma: Clinico-Pathologic, Phenotypic and Cytogenetic Analysis of 92 Adult Patients. Leukemia, 21, 340-350.

https://doi.org/10.1038/sj.leu.2404491

[2] Ohanian, M., Faderl, S., Ravandi, F., Pemmaraju, N., Garcia-Manero, G., Cortes, J. and Estrov, Z. (2013) Is Acute Myeloid Leukemia a Liquid Tumor? International Journal of Cancer, 133, 534-543. https://doi.org/10.1002/ijc.28012

[3] Tsimberidou, A.M., Kantarjian, H.M., Wen, S., Keating, M.J., O’Brien, S., Brandt, M., Pierce, S., Freireich, E.J., Medeiros, L.J. and Estey, E. (2008) Myeloid Sarcoma Is Associated with Superior Event-Free Survival and Overall Survival Compared with Acute Myeloid Leukemia. Cancer, 113, 1370-1378.

https://doi.org/10.1002/cncr.23691

[4] Neiman, R.S., Barcos, M., Berard, C., Bonner, H., Mann, R., Rydell, R.E. and Bennett, J.M. (1981) Granulocytic Sarcoma: A Clinicopathologic Study of 61 Biopsied Cases. Cancer, 48, 1426-1437.

https://doi.org/10.1002/1097-0142(19810915)48:6<1426::AID-CNCR2820480626>3. $\underline{0 . \mathrm{CO} ; 2-\mathrm{G}}$

[5] Shimizu, H., Saitoh, T., Hatsumi, N., Takada, S., Yokohama, A., Handa, H., Jimbo, T., Sakura, T., Tsukamoto, N., Murakami, H., Miyawaki, S. and Nojima, Y. (2012) Clinical Significance of Granulocytic Sarcoma in Adult Patients with Acute Myeloid Leukemia. Cancer Science, 103, 1513-1517. https://doi.org/10.1111/j.1349-7006.2012.02324.x

[6] Muss, H.B. and Moloney, W.C. (1973) Chloroma and Other Myeloblastic Tumors. Blood, 42, 721-728.

[7] Bakst, R., Wolden, S. and Yahalom, J. (2012) Radiation Therapy for Chloroma (Granulocytic Sarcoma). International Journal of Radiation Oncology, Biology, Physics, 
82, 1816-1822. https://doi.org/10.1016/j.ijrobp.2011.02.057

[8] Isshiki, Y., Ohwada, C., Togasaki, E., Shimizu, R., Hasegawa, N., Yamazaki, A., Sugita, Y., Kawaguchi, T., Tsukamoto, S., Sakai, S., Takeda, Y., Takeuchi, M., Sakaida, E., Shimizu, N., Ota, S., Yokote, K., Iseki, T. and Nakaseko, C. (2014) Acute Myeloid Leukemia Concurrent with Spinal Epidural Extramedullary Myeloid Sarcoma Accompanied by a High CD25 Expression and the FLT3-ITD Mutation. Internal Medicine, 53, 1159-1164. https://doi.org/10.2169/internalmedicine.53.1137

[9] Joseph, J.R., Wilkinson, D.A., Bailey, N.G., Lieberman, A.P., Tsien, C.I. and Orringer, D.A. (2015) Aggressive Myeloid Sarcoma Causing Recurrent Spinal Cord Compression. World Neurosurgery, 84, 866.e7-866.e10. https://doi.org/10.1016/j.wneu.2015.04.017

[10] Landis, D.M. and Aboulafia, D.M. (2003) Granulocytic Sarcoma: An Unusual Complication of Aleukemic Myeloid Leukemia Causing Spinal Cord Compression. A Case Report and Literature Review. Leukemia \& Lymphoma, 44, 1753-1760. https://doi.org/10.1080/1042819031000104051

[11] Takeda, M., Yamaguchi, S., Eguchi, K., Kajiume, T., Nishimura, S., Kobayashi, M. and Kurisu, K. (2009) Spinal Epidural Granulocytic Sarcoma in a Child Precedent to Clinical Manifestation of Acute Myeloid Lymphoma: Case Report. Neurologia Medico-Chirurgica (Tokyo), 49, 221-224. https://doi.org/10.2176/nmc.49.221

[12] Kook, H., Hwang, T.J., Choe, K., Yang, D.W., Nam, J.H. and Park, C.S. (1992) Spinal Epidural Granulocytic Sarcoma Preceding Acute Myelogenous Leukemia. Journal of Korean Medical Science, 7, 291-296. https://doi.org/10.3346/jkms.1992.7.3.291

[13] Wilhyde, D.E., Jane, J.A. and Mullan, S. (1963) Spinal Epidural Leukemia. The American Journal of Medicine, 34, 281-287.

https://doi.org/10.1016/0002-9343(63)90063-9

[14] Wodzinski, M.A., Collin, R., Winfield, D.A., Dalton, A. and Lawrence, A.C. (1988) Epidural Granulocytic Sarcoma in Acute Myeloid Leukemia with 8;21 Translocation. Cancer, 62, 1299-1300.

https://doi.org/10.1002/1097-0142(19881001)62:7<1299::AID-CNCR2820620709>3. $\underline{0 . \mathrm{CO} ; 2-\mathrm{T}}$

[15] Patchell, R.A., Tibbs, P.A., Regine, W.F., Payne, R., Saris, S., Kryscio, R.J., Mohiuddin, M. and Young, B. (2005) Direct Decompressive Surgical Resection in the Treatment of Spinal Cord Compression Caused by Metastatic Cancer: A Randomised Trial. The Lancet, 366, 643-648. https://doi.org/10.1016/S0140-6736(05)66954-1

[16] Seok, J.H., Park, J., Kim, S.K., Choi, J.E. and Kim, C.C. (2010) Granulocytic Sarcoma of the Spine: MRI and Clinical Review. American Journal of Roentgenology, 194, 485-489. https://doi.org/10.2214/AJR.09.3086

[17] Avni, B., Rund, D., Levin, M., Grisariu, S., Ben-Yehuda, D., Bar-Cohen, S. and Paltiel, O. (2012) Clinical Implications of Acute Myeloid Leukemia Presenting as Myeloid Sarcoma. Hematological Oncology, 30, 34-40. https://doi.org/10.1002/hon.994

[18] Yilmaz, A.F., Saydam, G., Sahin, F. and Baran, Y. (2013) Granulocytic Sarcoma: A Systematic Review. American Journal of Blood Research, 3, 265-270. 